Cinémas

Revue d'études cinématographiques

Journal of Film Studies

\title{
Présentation. L’ascension du cinéma moderne brésilien
}

\section{Hudson Moura}

Volume 22, numéro 1, automne 2011

Trajectoires contemporaines du cinéma brésilien

URI : https://id.erudit.org/iderudit/1005802ar

DOI : https://doi.org/10.7202/1005802ar

Aller au sommaire du numéro

Éditeur(s)

Cinémas

ISSN

1181-6945 (imprimé)

1705-6500 (numérique)

Découvrir la revue

Citer ce document

Moura, H. (2011). Présentation. L’ascension du cinéma moderne brésilien.

Cinémas, 22(1), 7-39. https://doi.org/10.7202/1005802ar d'utilisation que vous pouvez consulter en ligne.

https://apropos.erudit.org/fr/usagers/politique-dutilisation/ 


\section{Présentation. L'ascension du cinéma moderne brésilien}

\section{Hudson Moura}

Le cinéma brésilien de la période située entre 1995 et 2002 est souvent reconnu comme le cinéma de la Reprise (Cinema da Retomada). Ce qualificatif est controversé puisque les chercheurs et les cinéastes ne parviennent pas à un consensus sur ce que le mot «reprise" veut exactement dire, à savoir s'il signifie simplement la relance de la production cinématographique ou s'il représente un "renouveau", comme dans un mouvement esthético-social tel que le Cinema Novo des années 1960, ou encore s'il s'inscrit en quelque sorte dans la continuité de celui-ci. Quinze ans après cette extraordinaire remontée du cinéma brésilien, on doit pourtant repenser ce terme et se demander quelle "reprise» ce cinéma a été capable de surmonter et ce qu'il est resté de cette entreprise. En analysant la trajectoire contemporaine du cinéma brésilien, les articles réunis dans le présent numéro de la revue Cinémas cherchent aussi à entrevoir vers quel chemin esthético-narratif il s'oriente.

Depuis le succès mondial de La Cité de Dieu (Cidade de Deus) en 2002, le cinéma brésilien semble avoir atteint sa maturité sur le marché interne. Cependant, on voit avec beaucoup de préoccupation la monopolisation du marché par le conglomérat médiatique Globo. Au cours des quinze dernières années, dixsept films produits ou mis en marché par Globo Filmes ont fait partie des vingt films ayant récolté les plus grosses recettes au box-office. Troupe d'Élite 2 (Tropa de Elite 2, José Padilha, 2010), coproduit par Globo Filmes, a battu tous les records au box-office. Jusqu'en décembre 2010, le film a été vu par plus de 11 millions de Brésiliens, avec une recette de 66,2 millions de dollars ${ }^{1}$. Nous dirigeons-nous vers l'uniformisation de la production cinématographique?

En considérant l'importance des avancées théoriques et de la production du cinéma brésilien récent, je tenterai, dans la 
présentation de ce numéro de Cinémas, de dresser le bilan de l'histoire de la cinématographie brésilienne à travers ses éléments les plus marquants. Ce que l'on constate, c'est que le mouvement du Cinema Novo est devenu la référence "esthéticonarrative» pour les réalisateurs du cinéma contemporain, du moins selon la critique, principalement en ce qui concerne le retour de la notion d'identité nationale. La question de l'influence de la tradition critique dans la production et la réception des films ainsi que la quête de réalisme du cinéma fictionnel, par opposition à l'engagement esthétique et expérimental du cinéma documentaire, seront aussi examinées. Alors que le cinéma fictionnel tente de se rapprocher de l'univers naturaliste ou historique télévisuel pour attirer le public et intéresser le marché des films, le cinéma documentaire se distancie de ce procédé "facile», basé sur un récit «classique» et linéaire, pour adopter une formule plus expérimentale en utilisant des technologies de nature à soutenir les propos qu'il véhicule et en développant même une nouvelle esthétique pour filmer ce moment précis du cinéma brésilien — d'une façon urgente, comme le disent certains cinéastes.

\section{L'enchaînement d'une lente agonie?}

L'histoire récente du cinéma brésilien a été fortement marquée par l'ascension du régime démocratique du Brésil, après l'hégémonie de la dictature militaire, dont le règne a duré vingt ans (1964-1984). Les derniers événements historiques et politiques, principalement l'avènement du néolibéralisme, ont déterminé le cours de l'histoire et ont eu un impact important sur le cinéma brésilien - un reflet de ce qui s'est passé aussi dans d'autres pays comme l'Argentine et le Mexique. Le plan économique de mars 1990 du nouveau gouvernement du président Fernando Collor de Mello a profondément bousculé la vie du peuple brésilien. Il a suspendu toutes les formes d'aide financière qui, à l'époque, soutenaient tant bien que mal la production cinématographique, entraînant la fermeture de plusieurs agences, dont la traditionnelle Embrafilme, principale source de subvention. Subséquemment, le Concine (Conseil national du cinéma), la Fondation brésilienne du cinéma et le ministère de 
la Culture (réduit à un secrétariat) furent fermés et, enfin, les lois visant à encourager la production de films et la régulation du marché furent suspendues. Même les institutions chargées de produire des statistiques sur le cinéma au Brésil furent écartées. Le résultat de ces mesures sur la production cinématographique fut catastrophique, la moyenne chuta à un film par année, occupant $0,05 \%$ du marché intérieur.

Après le changement de gouvernement en 1994, et compte tenu du vent de néolibéralisme qui soufflait sur le pays, des lois spéciales furent adoptées, offrant des subventions à la réalisation de nouveaux films et des crédits d'impôt à des entreprises privées qui investissaient dans la production audiovisuelle locale. Les six années suivantes, la Loi de l'audiovisuel autorisait la production de près de 200 longs métrages, ce qui a permis au cinéma brésilien de connaître l'une des plus remarquables renaissances en Amérique latine. Malgré ce boom euphorique dans les champs de la réalisation et de la production, la distribution et la projection des films constituaient toujours une entrave au bon fonctionnement des choses, les lois gouvernementales ne prévoyant presque aucune aide financière dans ces domaines. Les films n'arrivaient pas à atteindre le public à cause d'un système de distribution contrôlé par des entreprises privées, souvent engagées auprès de distributeurs de films américains à gros budget. Les distributeurs se méfiaient des films brésiliens à cause de la mauvaise réputation du cinéma national qui, à l'époque, était connu pour être soit très intellectuel — du style du Cinema Novo, pas rentable —, soit très chargé "sexuellement", comme la pornochanchada (comédies érotiques à succès dans les années 1970), ce qui ne convenait pas au public familial. De son côté, le public aussi repoussait le cinéma local en se plaignant de sa mauvaise qualité artistique et technique (on arguait que le son, surtout, était incompréhensible ${ }^{2}$ ), qu'il comparait à la qualité des films étrangers ou des téléromans et séries télévisuelles.

Il a fallu une vedette de la télévision et de la pornochanchada pour briser la méfiance du public, de même que le monopole des distributeurs. L'actrice Carla Camurati, réalisatrice novice de longs métrages, dans une audacieuse et donquichottesque stratégie de distribution, voyagera avec quatre copies de son premier 
film et s'occupera personnellement de sa projection aux quatre coins du pays. Il s'avère que la comédie d'époque Carlota Joaquina, princesse du Brésil (Carlota Joaquina, princesa do Brazil) fera, en 1995, plus d'un million d'entrées et marquera le début d'une ère nouvelle pour le cinéma contemporain brésilien. Le film présente une vision satirique de la cour royale portugaise lorsque, sous l'emprise des Anglais, elle est obligée de fuir l'Europe et transfere provisoirement son royaume au Brésil, à l'époque des menaces d'invasion par le gouvernement français de Napoléon, en 1808. Certes, le film réunissait certains attributs tout aussi populaires que créatifs: des acteurs télévisuels connus et une rigoureuse recherche historique - permettant de saupoudrer le scénario de détails embarrassants et méconnus, de nature parfois coquine, sur la famille royale - associés à une réalisation précise et inventive s'appuyant sur un budget très modeste.

À la même époque fut lancé Terre lointaine (Terra estrangeira), réalisé par Walter Salles et Daniela Thomas, qui revint de Paris avec un prix du public. Ce film a également fait figure, pour d'autres raisons, d'emblème du renouveau du cinéma brésilien. Avec une allure documentaire présentant un caractère d'urgence, Terre lointaine présentait l'une des périodes économiques les plus sombres d'un passé très récent de l'histoire du pays, qui eut justement lieu sous le mandat du président Collor de Mello, qui venait tout juste de saboter la société brésilienne. Dans ce film, Manuela meurt à la suite de l'annonce faite par le gouvernement du gel de l'argent de tous les Brésiliens et, par conséquent, de ses propres économies. Après la mort de sa mère, Paco, un jeune étudiant sans argent et sans famille, saute sur la première occasion (comme plusieurs Brésiliens à l'époque) pour quitter le pays: il accepte de faire de la contrebande de diamants et se retrouve coincé dans la communauté naissante des exilés économiques brésiliens à Lisbonne, au Portugal. Le succès du film, autant auprès du public que de la critique, est dû, selon ses réalisateurs, à l'urgence de réagir à la situation décrite. D'ailleurs, c'est une espèce de reconstitution de l'histoire qu'on verra défiler sur les écrans tout au long de cette première période du cinéma de la Reprise. 


\section{L'héritage cinématographique}

Un des aspects du cinéma de la Reprise les plus critiqués et analysés à l'époque était l'utilisation, par quelques-uns des cinéastes associés à ce courant, des lieux mythico-symboliques du Cinema Novo: le sertão et la favela. Plusieurs chercheurs et critiques de cinéma ont aussi établi ou même "forcé » une comparaison entre la production récente des films et le mouvement le plus politisé de l'histoire (Oricchio 2003, Nagib 2002 et 2007). Le cinéma de la Reprise semble en effet refléter cette pensée politique, mais à travers un dialogue plutôt biaisé — ou inversé - avec le Cinema Novo, davantage qu'avec le Cinéma marginal. Cela constitue une nette différence si l'on compare ce cinéma avec la production récente de films, où ce sont plutôt le conformisme esthétique et le traitement "classique» des sujets, alliés à une quête d'identité nationale, qui prennent la place (Bentes 2003, Senra 2002). Mais on ignore la source culturelle du sertão et de la favela dans le cinéma de la Reprise, car ils servent plutôt de référence médiatique, influencée fortement par des images non seulement immortalisées par le Cinema Novo, mais aussi présentes dans la culture populaire de masse.

D'après Salles Gomes (1987) et Xavier (2001c), ce sont Nelson Pereira dos Santos dans les années 1950 et ensuite le Cinema Novo et le Cinéma marginal qui ont donné sa forme au cinéma moderne au Brésil. Une modernité ancrée dans la convergence de la politique des auteurs, les productions à petit budget et le renouvellement esthétique.

Le Cinema Novo a explosé dans les années 1960 sous la tutelle d'une nouvelle génération de cinéphiles de formation universitaire, engagés dans la création d'un cinéma "authentiquement" brésilien. Le mouvement a officiellement été lancé en 1963 avec les films Le dieu noir et le diable blond (Deus e o diabo na terra do sol) de Glauber Rocha, Les fusils (Os fuzis) de Ruy Guerra et Sécheresse (Vidas secas) de Nelson Pereira dos Santos. La caractéristique principale du mouvement était d'allier le cinéma aux questions sociales et à la politique de gauche et, dans le cas de Rocha, aux contes populaires.

Les cinéastes du Cinema Novo ont associé une esthétique moderne à un discours politique radical afin d'exposer les 
réalités de la pauvreté, le sous-développement et l'exploitation du peuple brésilien. Se réclamant des deux manifestes écrits par Glauber Rocha - L'esthétique de la faim et L'esthétique du rêve - le mouvement a connu de grands succès partout dans le monde auprès de la critique spécialisée, particulièrement avec des films comme Terre en transe (Terra em transe, 1967) et Antonio das Mortes ( $O$ dragáo da maldade contra o santo guerreiro, 1969) de Glauber Rocha, Le défi (O desafio, 1965) de Paulo César Saraceni, Le prêtre et la jeune fille (O padre e a moça, 1966) de Joaquim Pedro de Andrade, Ganga Zumba (1963) de Carlos Diegues, La morte (A falecida, 1965) de Leon Hirszman, Les dieux et les morts (Os deuses e os mortos, 1970) de Ruy Guerra et Faim d'amour (Fome de amor, 1968) de Nelson Pereira dos Santos. Ils ont créé l'un des plus actifs et inventifs mouvements du cinéma moderne mondial, qui a stimulé le questionnement autour du rôle des cinémas nationaux dans le "tiers-monde». Influencé par le néoréalisme et la Nouvelle Vague, le Cinema Novo est connu pour avoir insisté sur le caractère politique du cinéma ${ }^{3}$, comme l'a déclaré Carlos Diegues aux journaux de l'époque: "Nous faisions des films politiques quand la Nouvelle Vague était encore en train de parler de l'amour non partagé ${ }^{4}$." Un mouvement du cinéma politique aussi présent dans les pays voisins, instauré par Fernando Solanas et Octavio Gettino en Argentine avec leur Troisième cinéma, ou encore avec le Cinéma imparfait de Julio García Espinosa à Cuba.

Malgré les efforts des cinémanovistes, le public brésilien a jugé leurs films très intellectuels et "hermétiques». Une partie de cette incompréhension est due à la censure d'État sous le régime militaire. Le Cinema Novo a trouvé sa voix de plus en plus dans les formes les plus déguisées de l'allégorie, de la métaphore, du symbolisme et de la satire. À la fin des années 1960, quand la répression et la censure militaires devinrent trop sévères, des réalisateurs comme Glauber Rocha fuiront en exil et l'énergie du mouvement s'étiolera. D'autres sous-genres moins contestataires ou politiques vont se créer, comme le tropicalisme de Joaquim Pedro de Andrade (Macunaïma/Macunaima, 1969), de Walter Lima Jr. (Brésil année 2000/Brasil ano 2000, 1969) et de Nelson Pereira dos Santos (Comme il était bon mon petit 
Français/Como era gostoso meu francês, 1971), ou l'adaptation littéraire, genre exploité par Leon Hirszman dans São Bernardo (1972), par exemple. Certains réalisateurs vont continuer de faire des films en exploitant plus tard le genre érotique, comme Joaquim Pedro de Andrade (Contes érotiques/Contos eróticos, 1977), Arnaldo Jabor (Toute nudité sera châtiée/Toda nudez será castigada, 1973) et Carlos Diegues (Xica da Silva, 1976), pour échapper à la censure de la critique sociale sous le régime dictatorial.

Le Cinéma marginal va prendre la relève du cinéma contestataire en ayant recours à la "transgression » et à l'invention esthétique, qu'il alliera souvent à un contenu socialement et «sexuellement» violent et choquant. L'intention des réalisateurs était ici, de toute manière, de rompre le contrat tacite assurant un rapport harmonieux entre le spectateur et le film et, surtout, de s'écarter de l'idée d'identité nationale fortement soutenue par le Cinema Novo (Xavier 2001c). Les cinéastes les plus connus de ce "mouvement» sont Rogério Sganzerla (Le bandit de la lumière rouge/O bandido da luz vermelha, 1968), Andrea Tonacci (Bang, Bang, 1969), José Mojica Marins (alias Zé do Caixão) (À minuit je posséderai ton âmelÀ meia noite levarei sua alma, 1964) et Júlio Bressane (Il a tué sa famille et est allé au cinéma/Matou a familia e foi ao cinema, 1969). Le mot "marginal" attribué à ces films produits sous la forte censure du régime militaire entre 1968 et 1973 ne fait pas pour autant consensus. Le témoignage de l'artiste Lygia Pape définit bien l'esprit du terme: "Marginal a été l'acte révolutionnaire de l'invention, une nouvelle réalité, le monde comme changement, l'erreur comme aventure et découverte de la liberté... l'anti-film»(Pape 2004, s. p.). Ces films sont aussi connus sous ces désignations: films expérimentaux, alternatifs, cinéma de l'invention, cinéma de poésie, cinéma underground - ce qui démontre la variété et les différences de styles et d'approches des sujets traités. Xavier (2001b, p. 5) convient qu'il est ardu de le concevoir comme mouvement, car il présente une riche

diversité de postures, qui va au-delà des traits communs, des déclarations incisives prônant le recours à un budget minimal et défendant un cinéma d'auteur, agressif, qui ne fait aucune 
concession et est prêt à heurter par la texture de l'image, la violence des gestes et le grotesque des visages qu'il fait défiler.

Les particularités techniques ou esthétiques de ce cinéma vont fortement influencer les réalisateurs de comédies érotiques associés à la pornochanchada.

Il est vrai que ce cinéma populaire érotique se définit principalement contre la politisation extrême et l'hermétisme intellectuel du Cinema Novo. Ces films sont aussi connus comme provenant de la "Boca do Lixo", un quartier mal famé du centre-ville de São Paulo, où étaient situées la majorité des maisons de production du cinéma marginal et commercial dans les années 1970. À cette époque, la production nationale comptait 80 films par année, dont $40 \%$ étaient réalisés dans ce quartier, incluant des films érotiques, policiers, expérimentaux, des drames et des comédies. La pornochanchada devient alors l'«industrie responsable» des films les plus populaires de l'histoire du cinéma brésilien. Notons que ce terme, pornochanchada, vient de l'assemblage de deux genres: le cinéma érotique (porno) et les comédies musicales (chanchadas). Ces dernières ont connu un énorme succès dans les années 1940-1950 en parodiant avec beaucoup d'humour les films hollywoodiens; leur pouvoir d'attraction peut aussi être lié au fait qu'on y avait recours à des humoristes et à des chanteurs popularisés par la radio. La grande majorité de ces films ont été produits à Rio de Janeiro dans les studios Cinédia et Atlântida, où ils étaient soumis à un style de production à l' " américaine».

Ainsi que les chanchadas, les pornochanchadas réalisées, entre autres, par David Cardoso (La nuit des fétiches/A noite das taras, 1980), Ody Fraga (La réforme des dépravés/Reformatório das depravadas, 1978), Jean Garret (L'île du désir/A ilha do desejo, 1975), Silvio de Abreu (Femme objet/Mulher objeto, 1980) et Fauzi Mansur (La nuit des femelles/A noite das fêmeas, 1976) ont fait fureur au box-office. D'autres cinéastes dotés d'un style d'une grande originalité vont parvenir à créer des œuvres à caractère personnel dans le contexte commercial d'un cinéma populaire, comme Carlos Reichenbach (Lilian M., 1975), Francisco Ramalho Jr. (À fleur de peaulÀ flor da pele, 1976), João 
Batista de Andrade (Doramundo, 1978), Hector Babenco (Lucio Flavio: le passager de l'agonie/Lúcio Flávio: O passageiro da agonia, 1977 et Pixote: la loi du plus faible/Pixote: A lei do mais fraco, 1980) et Bruno Barreto (Dona Flor et ses deux maris/Dona Flor e seus dois maridos, 1978).

Selon Xavier (2001c), le meilleur du cinéma brésilien des années 1970 a gravité autour de drames familiaux, de récits relatant des expériences où les protagonistes tombent dans la décadence, de crises aiguës vécues par des personnages frustrés dont les efforts pour améliorer leur sort ont échoué, ou de pérégrinations catastrophiques de pauvres comme de riches qui se sont terminées dans le malheur. En effet, les films de cette époque ont été contaminés par un sentiment d'impuissance que le critique de cinéma et écrivain Paulo Emílio Salles Gomes (1987, p. 12) a résumé dans la formule suivante: le sous-développement du cinéma brésilien n'est pas une phase, mais un état permanent.

\section{Cinéma sous-développé : tout est étranger}

Paulo Emílio Salles Gomes, dans son article «Le cinéma: trajectoire dans le sous-développement", fait une analyse comparative aiguë du sous-développement du cinéma mondial, incluant le cinéma brésilien. Comme chroniqueur de son temps, Salles Gomes voyait clairement l'importance du cinéma comme art national; il dévoile le Brésil à travers le cinéma et la condition du cinéma par une analyse profonde du pays, dans une des périodes les plus sombres de son histoire. La réalité brésilienne est révélée dans les images qui défilent sur l'écran et qui la médiatisent, permettant au critique de définir plus aisément les notions de "Brésil ", "brésilien", "public», "peuple» et "sousdéveloppement». Ce n'est pas un hasard si Salles Gomes est l'auteur d'une des phrases les plus régionalistes du cinéma brésilien : "Même le pire film brésilien nous en dit plus que le meilleur film étranger ${ }^{5}$." Son but était surtout de montrer ô combien le peuple s'identifie et établit un lien étroit avec les images que lui-même crée.

$\mathrm{Si}$, auparavant, il était important de mettre l'accent sur la finesse et l'intelligence des écrits de Salles Gomes, aujourd'hui il 
est essentiel de souligner son rôle d'historien et de sociologue du Brésil, et de ne pas le considérer seulement comme l'un des plus importants et influents critiques entre les années 1950 et 1970. Ainsi, durant plus de trente ans, Salles Gomes a rapporté et commenté, avec beaucoup de justesse, les faits divers du Brésil tels que montrés au cinéma. Il a révélé la grande capacité du cinéma de faire entrevoir une image non homogène du pays à travers ses histoires.

Selon Salles Gomes, le cinéma est le médiateur (ou média) qui ouvre l'accès au monde. Le cinéma suscite à chaque instant des réflexions sur l'homme et la société. Le critique a de la sorte dévoilé l'histoire du Brésil en dialoguant avec des films comme Le sang mineiro (Sangue mineiro, 1930) de Humberto Mauro, Sans peur, sans pitié (O cangaceiro, 1953) de Lima Barreto, ou avec les films de la nouvelle génération de cinéastes du Cinema Novo ou du Cinéma marginal. Salles Gomes a même été reconnu par les cinémanovistes comme le père intellectuel du Cinema Novo.

Pour le cinéma brésilien, le sous-développement n'est pas une étape, un stade, mais une condition, affirme Salles Gomes. Les cinémas américain, japonais, européen en général, n’ont jamais été sous-développés, tandis que les cinémas hindou, arabe ou brésilien n'ont jamais cessé de l'être.

Le Brésil est une extension de l'Occident, dont le peuple n'a jamais été, à proprement parler, "occupé»: «Nous ne sommes ni Européens ni Nord-Américains, mais dépourvus d'une culture originale; rien ne nous est étranger car tout l'est. Nous nous construisons péniblement nous-mêmes dans la dialectique raréfiée entre le "non-être" et l'"être-autre" "(Salles Gomes 1987 , p. 12). Le film brésilien, toujours selon le critique, participe à cette construction identitaire et la modifie à travers son " incompétence créatrice au copiage».

Salles Gomes insiste sur le fait que la création du cinéma était étrangère au peuple sous-développé et que celui-ci n'est toujours pas parvenu à produire de films selon une technique qui lui serait propre, pas plus qu'il n'est parvenu à produire un discours national imagé. Les comédies et les films musicaux de l'Atlântida, ainsi que les drames «pompeux» du studio Vera Cruz, qui sont 
des parodies ou des «copies» des films hollywoodiens, en sont les principaux exemples. Mais les films de la chanchada ont été les seuls à être "parfaitement adéquats et soumis à notre condition générale sous-développée» (1987, p. 15). Par contre, le Cinema Novo a été l'expression culturelle la plus raffinée d'un très grand moment cinématographique national. Il a su explorer le sousdéveloppement du peuple et a transposé à l'écran la condition de ce peuple colonisé: "Adieu le héros chômeur de la chanchada, il est remplacé par le travailleur; mais lors des projections, les occupés se trouvaient plus présents sur l'écran que dans les salles" (1987, p. 14). Le Cinema Novo, comme mouvement intellectuel et politique issu des universités et de la presse spécialisée, n’a pas atteint la grande majorité du peuple brésilien, comme ce fut le cas des films de la chanchada. Certes, l'écartèlement du Cinema Novo est plutôt dû à la pression de la politique intérieure. Malgré cela, pour la première fois, on a pu observer une forte réaction de la part des cinéastes indépendants en vue de parvenir à une définition du cinéma national. Cette expérience a finalement permis au concept de cinéma brésilien de voir le jour et on ressent toujours la forte influence qu'a eue le Cinema Novo auprès de la société et du cinéma contemporains.

Le concept de "cinéma national ", tellement cher aux réalisateurs et aux chercheurs en cinéma, n'est pas fixe, mais toujours en évolution et en changement, répondant aux besoins de tel ou tel cinéma et de son public immédiat à un moment donné de son histoire. Et pourtant, durant la Reprise, la quête d'une définition propre au «cinéma national» était tellement importante qu'on a eu l'impression que les réalisateurs étaient animés d'un désir "aveugle» que leurs films soient acceptés par le public et d'une volonté féroce de développer une cinématographie qui pouvait répondre à la mondialisation de la production et du marché.

Nagib (2007, p. xviii) souligne que le début des années 1990, sur le plan socioculturel, peut être considéré comme le moment marquant l'apparition des cultural studies - cette époque a également accordé une plus grande place aux films ethniques, aux minorités sexuelles, et a été caractérisée par le retour du cinéma engagé - , de la politique de gauche du political correctness, de la 
prolifération des festivals de films et des espaces alternatifs. Tout cela a favorisé l'ascension d'une nouvelle génération de cinéastes ainsi que l'intérêt grandissant du public pour les particularités locales et nationales, en réaction à l'homogénéisation menaçante de la mondialisation.

Le cinéma de la Reprise représente sans conteste le quatrième plus important moment du cinéma brésilien, après la «belle époque» des années 1930, la chanchada des années 1940 et le Cinema Novo des années 1960. Les cinéastes de ce mouvement ont su rallier le public autour du débat national et des thèmes qui y étaient liés: la question de l'appartenance, la critique historique, la popularisation du cinéma, la régionalisation de la production et la reconnaissance internationale de ses artisans locaux. Par contre, le sous-développement dans la sphère politique et dans le domaine de la production cinématographique n'est plus central dans la réalisation des films, puisque ceux-ci se sont fait remarquer par leur haute qualité technique et par leurs modes narratifs (histoires, scénarios) plutôt standard. À titre de comparaison, il semble important de faire un parallèle avec les cinémas de la Chine et des Philippines, comme Salles Gomes l'a fait autrefois avec les cinémas hindou et arabe. Le cinéma actuel de la sixième génération en Chine, qui souffre d'une rigoureuse censure gouvernementale, a forcé ses réalisateurs à assurer autrement sa «survivance» dans les années 1990. Ils ont dû solliciter le soutien financier des producteurs internationaux du Japon et de la France, et profiter du faible coût du numérique et des marchés «alternatifs» des DVD pirates pour produire et distribuer leurs films. Quant aux cinéastes des Philippines, ils ont su développer un lien fort entre leurs thématiques locales — les mélodrames télévisuels - en ayant recours aux nouvelles technologies de la vidéo numérique pour reconstruire leur cinéma national.

La préoccupation des cinéastes brésiliens des années 1990, qui désiraient créer un portrait du pays, est manifeste. Ils reprennent le débat de l'identité nationale et les décors mythiques de l'histoire du cinéma : sertão et favela — une auto-référentialité importante pour rétablir une notion que le cinéma brésilien avait perdue durant la période suivant la dictature: sa propre identité. Ce n'était pas seulement le nombre de productions qui était 
réduit à zéro ${ }^{6}$ au début des années 1990 , on avait aussi perdu l'estime de soi, de même que ses objectifs; par conséquent, la télévision, soumise aux intérêts gouvernementaux, a remplacé le cinéma. Le peuple, ou l' "occupé», n'apparaît ni sur l'écran ni dans les salles de cinéma. Le cinéma de la Reprise a atteint le public de classe moyenne ou d'une classe ayant de fortes aspirations d'ascension sociale habituée aux histoires télévisuelles et du cinéma populaire propulsé par des sous-produits de la chaîne télévisée Rede Globo, comme Trapalhóes et Xuxa.

\section{Xavier : entre archaïsme et modernité}

Aujourd'hui, on peut presque comparer l'influence de Salles Gomes sur la culture cinématographique brésilienne à celle de l'essayiste et professeur de cinéma à la Universidade de São Paulo, Ismail Xavier. Depuis la publication de son étude approfondie sur le cinéma de Glauber Rocha dans les années 1980 (voir Xavier 1983, traduit en français en 2009, et Xavier 1993), Xavier s'est fait remarquer pour son analyse exhaustive du concept de "cinéma national». Xavier impose un style et une forme de pensée au cinéma directement influencés par la tradition critique à laquelle adhèrent ses professeurs - Salles Gomes et le sociologue Antônio Cândido - , où les concepts de cinéma et de Brésil se confondent, en rapprochant l'esthétique cinématographique à des "structures sociales", approche s'apparentant à la tradition anglophone des cultural studies au cinéma. Certes, on doit à Xavier les analyses les plus fines et les plus pointues sur ce récent tournant du cinéma brésilien. Il a défini certains paradigmes, comme celui de la rencontre inattendue (Senra 2006; Moura 2008) et celui du ressentiment national ${ }^{7}$, à partir desquels il a analysé les dernières tendances du cinéma brésilien et les récurrences qu'on peut y observer.

Le thème de la rencontre inattendue, d'après les constatations de Xavier, figure dans une série de films de la Reprise, où la rencontre a lieu entre des personnages mal assortis ou avec l'étranger (Amélia d'Ana Carolina; Comment naissent les anges/Como nascem os anjos de Murilo Salles; Le bal parfumélBaile perfumado de Paulo Caldas et Lírio Ferreira; Cinéma, aspirines et vautours/ Cinema, aspirinas e urubus de Marcelo Gomes; Quatre jours en 
septembre/O que é isso, companheiro? de Bruno Barreto, entre autres). Dans ces films, il y a d'ores et déjà, en termes cinématographiques, "repositionnement» d'une certaine idée de la relation centre-périphérie: l'étranger est rationnel alors que les locaux sont des gens plongés dans un conflit irrationnel ou difficile à comprendre. Il s'agit d'une nouvelle façon d'étudier la relation entre colonisateurs et colonisés: marquée par un ressentiment mutuel, non résolu. La rancœur des personnages de ces films semble venir du passé; l'accumulation de leur colère explose dans la violence. Le ressentiment des riches n'est pas le même que celui des pauvres, mais il est présent dans toutes les classes sociales, ainsi qu'on peut entre autres le voir dans des films comme Chroniquement irréalisable (Cronicamente inviável) de Sérgio Bianchi, Coup de feu entre amis (Ação entre amigos) de Beto Brant, Un verre de rage (Um copo de cólera) d'Aluizio Abranches, Anahy de las misiones de Sérgio Silva et Le premier jour ( $O$ primeiro dia) de Walter Salles. Ces films manifestent l'absence d'un projet national, mais ils expriment aussi l'absence d'un horizon utopique. Dans le documentaire, ces rencontres inattendues ont encore plus de résonance puisque le cinéaste se présente comme un individu, un citoyen doté d'une histoire particulière. Il ne prétend pas au rôle de représentant, il ne vient pas avec un mandat. Le débat cible plutôt l'individu sur le plan personnel, non pas les structures sociales.

Dans Central do Brasil, film clé du cinéma de la Reprise, "le défilé de visages qui dictent des lettres est d'une densité rare dans le cinéma brésilien, ainsi que quelques images de ce parcours de migration en sens inverse qui établit un dialogue avec les films du Cinema Novo" (Xavier 2000a, p. 102). Cependant, Xavier voit dans ce film la répétition des pires clichés antiurbains, puisqu'une polarisation éthique entre l'archaïque et le moderne y est créée.

Toujours selon Xavier, le thème historique a été repris de façon fort intéressante par le cinéma contemporain, où l'on met souvent l'accent sur le sertão et où il y a là un dialogue conscient avec les traditions du cinéma lui-même. Ainsi, ce cinéma ne reprend pas les thèmes du Cinema Novo, puisqu'il dialogue avec le cinéma en tant que tel. "Le cinéma de fiction 
au Brésil a créé, en particulier dans les années 1960 et 1970, une forte tendance au discours politique totalitaire, orienté par les constats généraux par rapport à la situation du pays " (Xavier 2000a, p. 101). Ce discours, ou encore ce constat, n'est absolument pas présent dans le cinéma actuel, qui ne discute pas les structures sociales. Dans ses rapports avec la question de la violence, tant dans sa version rurale (le film de bandits: les cangaceiros) que dans sa version urbaine (le crime organisé, les échanges de tirs dans les bidonvilles, la question de l'enfant des rues), le cinéma contemporain crée un autre lien avec les réalités actuelles du Brésil.

Il n’y a plus de personnages «sacrifiés", comme les Lampião ou Corisco de Glauber Rocha, ou le Lúcio Flávio d'Hector Babenco, qui expriment leur violence dans une lutte contre le pouvoir établi, affirme Xavier. Dans les films récents, le méchant est seulement un criminel ou un rancunier en quête de vengeance. "Nous vivons dans une période où l'on tente d'éviter la discussion sur les structures sociales, où il est dit que les problèmes, les particularités de certains aspects politiques de ce caractère national, quel qu'il soit, pèsent sur les consciences, et non sur les structures" (Xavier 2000b, p. 9). L'ordre du jour prépondérant veut que chacun prenne soin de soi et de ses biens, non seulement à cause de ce qui se passe uniquement au Brésil, mais en raison du cadre mondial. La politique, sur la scène internationale, ne prétend pas résoudre les problèmes mondiaux, mais cherche des solutions individuelles et localisées.

Dans le cinéma contemporain, sont absents deux éléments qui étaient visibles dans les films situés dans le sertão : la violence et la religion. Dans la tradition de la culture brésilienne:

Le sertão vaut comme unité et privilégie l'analyse des formes, comme l'identité, notion qui a été conçue par le régionalisme du $\mathrm{XIX}^{e}$ siècle, dans un processus qui s'est révélé progressif: une "logique interne», des particularités culturelles et des modes de distribution du pouvoir à partir desquels on a commencé à explorer les potentialités de cette unité au-delà de la question régionale, comme un symbole ou une synthèse de totalités plus vastes (Xavier 2002, p. 10). 
Au fil des dernières années, constate Xavier, le cours de la culture et de la politique brésilienne a fait naître chez les cinéastes un sentiment de résignation devant la fin de l'utopie dans le cinéma moderne. Par conséquent, il y a un décalage entre l'image de soi du réalisateur, qui se préoccupe encore de la politique de l'identité nationale, et la nécessité de réaliser des films où ne se reflète pas cette politique, sinon à de rares exceptions près. Les porte-parole de la communauté se retrouvent aujourd'hui sur un terrain occupé par la rhétorique de la Rede Globo, avec sa version industrialisée et commercialisée du populaire national, dont sont imprégnés ses feuilletons et ses séries télévisuelles.

Parmi les mécanismes de production d'images, le film serait davantage en mesure que la télévision de créer une distance et de promouvoir la critique et la réflexion. Au Brésil, le cinéma, qui n'est pas un art de masse, cherche maintenant à exploiter une relation étroite avec la télévision. Mais cela est encore très timide, car il n'a pas suffisamment développé ses possibilités esthétiques ni même réussi à discuter à fond des problèmes de la société brésilienne.

\section{Mouvement ou style : de quelle reprise parlons-nous?}

Il est important de faire un bilan des écrits de Salles Gomes et de Xavier au fur et à mesure qu'ils guident les critiques et les analyses universitaires du cinéma au Brésil. En effet, l'expression "cinéma de la Reprise» a été créée par la critique et par les chercheurs, et non par les réalisateurs ou les producteurs.

Depuis les constats sur la modernité dans les films de Nelson Pereira dos Santos dans les années 1950, le cinéma brésilien, avec son esthétique néoréaliste et son engagement social, a emprunté une «trajectoire» tout à fait conforme à l'esprit de son temps. Mais, si le concept de modernité au cinéma, pour Salles Gomes (1987) et Xavier (2001c), est étroitement associé à l'image de l'auteur engagé répandue par le Cinema Novo, pour Kovács (2007), il se définit entre autres par les techniques et les formes stylistiques du cinéma d'art, ce qui signifie qu'il faut comprendre ce cinéma en fonction de ses attributs artistiques et de ses variations formelles. Dans ce sens, la modernité au 
cinéma doit être comprise par son avant-gardisme artistique plutôt que par ses "modernismes» universels. Ainsi, on met l'accent sur les avancements techniques et formels particuliers de chaque cinéma national, indépendamment de son époque.

Par contre, certains cinéastes refusent "même» de parler de mouvement dans le cinéma brésilien récent - qu'il s'agisse de celui de la «reprise» ou de quelque autre cinéma - car, selon eux, aucun groupe ne s'est réellement formé autour d'intérêts communs (politiques ou sociaux) ou d'une esthétique particulière. Malgré cela, l'utilisation de ce terme a été pratiquement généralisée et tous l'ont adopté. Il nous reste donc à nous demander quelle "reprise» a eu lieu durant la période visée. «Reprise» de la production? À ce moment, il s'agissait d'un phénomène purement économique, mais il semble que le terme veuille dire plus, puisque certaines particularités et tendances se répètent dans tous les films qu'on a classés sous ce vocable. En vérité, la "reprise" coïncide presque avec l'avènement de la mondialisation - que ce soit l'industrialisation de la production, l'ouverture du marché, le partage de l'information et de la technologie, la participation à de nombreux festivals internationaux, l'accès aux sources de financement étranger, etc. Du moins, c'est le moment où nous commençons à répondre à la mondialisation en termes cinématographiques. C'est aussi le moment où surgit une pléthore de questions.

La production cinématographique récente ne présente aucune rupture esthétique ou sociale avec l'histoire du cinéma brésilien, puisqu'il y a une sorte de continuité dans la tradition depuis les années 1940, où l'identité nationale et ses lieux mythiques sont privilégiés: le sertão du Nordeste et les favelas des grandes villes. Dès sa création, en 1941, le studio Atlântida s'attardait dans son manifeste à la nécessité de "filmer des sujets brésiliens, susceptibles de conférer à la réalité nationale une existence sur les écrans» (Galvão et Souza 1987, p. 76). Ce discours se répétait dans le manifeste de Rocha dans les années 1960 et dans les entrevues accordées par les réalisateurs de la Reprise, comme Walter Salles à l'époque du lancement de Central do Brasil (1998).

On se souvient d'un cinéma d'auteur proprement brésilien, associé au Cinema Novo, mais qui n’a pas nécessairement exercé 
une influence esthétique ou politique sur le cinéma de la Reprise. Fernando Meirelles, le réalisateur de La Cité de Dieu (2002), lors d'un débat ardent sur son film, a affirmé trouver Glauber Rocha ennuyant. À vrai dire, c'est une idée assez répandue, et qui l'était principalement à la fin des années 1980, alors que le cinéma subissait les pires moments d'une crise qui se manifestait au sein du public, mais aussi aux niveaux économique et créatif. D’ailleurs, c'est dans cet état de méfiance et de stérilité qu'a surgi la nouvelle génération d'artisans qui, comme Meirelles, ont dû aller travailler à la télévision et dans la publicité avant de commencer une carrière sur un plateau de tournage. Leur situation et leurs débuts en tant que professionnels étaient différents de ce qu'avaient connu les jeunes cinéphiles et universitaires du Cinema Novo. Par ailleurs, ils étaient moins convaincus de leurs positions politico-sociales et leur attitude était moins tranchée par rapport aux questions nationales populaires. Les cinéastes de la Reprise s'attardent aux mêmes sujets et aux mêmes thèmes que leurs prédécesseurs, ils filment les mêmes endroits, mais à partir d'un regard autre, qu'ils ne sont pas tenus d'orienter en vue d'exprimer une quelconque quête de transformation sociale ou de veiller à la construction d'une allégorie nationale, mais où la quête de l'individu a pris toute la place au détriment du récit.

Dans Carandiru (2003) d'Hector Babenco, l'histoire individuelle de quelques prisonniers circonscrit l'espace narratif sans nécessairement constituer le récit principal: le massacre de 111 prisonniers tués par la police à la suite d'une rébellion, un fait réel de l'histoire brésilienne récente. Un sujet et un univers bien connus de Babenco, qui a réalisé des films à succès comme Lúcio Flávio (1977) et Pixote (1980). Est-ce que cela représente une crise dans le traitement des histoires, un manque d'imagination ou simplement un désintérêt pour l'intrigue? Le massacre qui devrait être le point culminant du film ne devient qu'un simple événement qui se termine avec la mort de certains personnages. Le massacre n'est ni le point culminant ni la raison pour laquelle les histoires "individuelles» se forment; elles se construisent et s'achèvent par elles-mêmes, sans que pèse sur elles l'imminence de l'événement qui va se produire. Les 
personnages ne réagissent pas en fonction de cet événement et ils en sont encore moins directement la cause.

Le renouveau esthétique et structural de ce cinéma contemporain présentera plus d'affinités avec les séries télévisuelles et les téléromans (Hamburguer 2005). Cela est probablement dû au remplacement du discours idéologique sur l'identité nationale par la rhétorique du gagnant dans la bataille audiovisuelle menée par Globo (Xavier, 2003). Beaucoup de films, par ailleurs, ont actuellement tendance à reproduire les idées et le style de la téléfiction pour des raisons commerciales.

La vie peu ordinaire de Dona Linhares (Eu Tu Eles, 2000) d'Andrucha Waddington offre une image extrêmement bien «travaillée» du sertão, avec des vedettes de la télévision dont le charisme naturel s'ajoute à l'aspect comique du film. L'histoire du film n'est certes pas construite selon les critères du cinéma standard, où l'intrigue devient l'une des plus importantes forces motrices du scénario. Le film ne crée aucune attente et le spectateur suit simplement le déroulement de l'histoire. Les personnages se présentent bel et bien comme des "individus " à l'écran, non pas en fonction de l'histoire qui est racontée, mais plutôt en fonction de leur manière de vivre. Dans ce portrait du sertáo, le poids dramatique du récit s'estompe grâce à une photographie contrastée, à des couleurs chaudes et à une grande qualité technique.

Le sertão est dépeint ici d'une façon unique, puisqu'il n'est pas situé dans une région pauvre et dévastée par la sécheresse, où les conditions de vie soulèveraient inévitablement des questions d'ordre sociologique et culturel. Ce sertão provient de la médiation, des images créées et répandues depuis la création des films de cangaceiros dans les années 1950, ou depuis l'apparition de la littérature populaire de cordel. Le sertão, l'un des berceaux de l'histoire du Brésil, est aussi le lieu de naissance du Cinema Novo, qui a largement exploité ses dimensions humaines et géographiques. Bien que le sertáo soit le décor dans lequel reposent les histoires populaires de bandits et de prophètes, il n'y a dans l'approche du cinéma de la Reprise rien de sociologique ou d'idéologique dans l'utilisation de ce lieu, comparativement à l'usage qu'on en faisait dans les années 1960. Glauber Rocha et 
le groupe cinémanoviste constituent plutôt une référence imagée qu'une influence. Dans les films de la Reprise, le sertão sert plutôt à une expérience esthétique.

\section{Central do Brasil: en quête d'une identité}

Une quête des origines pour combler une absence est le thème de Central do Brasil (1998) de Walter Salles. Le début se déroule à Rio et le film se poursuit dans le sertão, où le réalisateur se met en quête des racines d'un peuple et d'un cinéma. Le film met à nu un pays difficile à montrer, et c'est cela le plus dérangeant. Il révèle, en même temps, une absence dans la cinématographie nationale: le pays lui-même, le visage de son peuple, une image qui serait engagée. Dans ce film, Salles essaie d'exhumer d'innombrables coutumes, habitudes, gestes, paroles, accents et histoires dont on ne faisait que rarement état dans les médias brésiliens. À partir de l'histoire d'un gamin qui recherche son père alors qu'il vient de perdre sa mère, le film raconte l'histoire du pays.

Central do Brasil permet un retour dans le sertão, qui représente ce genre d'endroit résistant à toute nouvelle avancée du progrès et où nous pouvons retrouver une certaine tradition orale, une certaine parole, perdue dans le temps, une histoire qui semble appartenir au passé, un certain peuple, plus "authentique», plus "vrai». Le film montre qu'il est primordial de retrouver ce Brésil "perdu», oublié; c'est la tâche que s'est donnée le cinéaste, reconquérir une tradition obsolète, démodée: c'est le sentiment de saudade enraciné dans ses images. Une claire nostalgie du Cinema Novo, comme si la production nationale avait été prise en otage pendant plus de trente ans, soit depuis l'accession des militaires au pouvoir en 1964. À travers des histoires privées, le film reprend des questionnements actuels : retrouver le collectif dans un style documentaire, réaliste, non dénué de sentimentalisme, de romantisme et, surtout, d'un certain humanisme. Deleuze (1985) a dit à propos du cinéma révolutionnaire de Glauber Rocha que, comme il n'y avait pas de peuple, il fallait l'inventer, lui donner corps, voix et âme. Donc, de prime abord, c'était l'absence de ce peuple que le cinéma glauberien montrait. Peut-être Salles essaie-t-il de 
reproduire cela, mais évidemment par des choix esthétiques et sur la base d'idéaux différents. Autrement dit, il essaie de trouver ce sujet authentique, cette culture dynamique qui ne serait pas encore touchée par les «mauvaises» habitudes d'un regard trop médiatisé. Le film ose opérer un retour aux origines nationales, même si le ton semble être trop sentimental et les idéaux, trop humanistes pour les goûts d'aujourd'hui. Quel regard porte-t-il sur le pays?

Le Brésil revisité par Central do Brasil est un pays de contrastes, dont la foi est l'unique voie de rédemption. Le mouvement des personnages dans la gare, sur l'autoroute, au milieu de la procession, se confond avec leur mouvement d'identification et de rejet mutuel. Le tour de force entre l'institutrice retraitée sous-employée et le gamin sans mère nous rapproche indirectement d'une vérité qui se trouve toujours à l'arrière-plan de l'immense, extrêmement beau, bien photographié et très coloré désert du sertão. Une réalité complètement différente de celle du Cinema Novo, dont la force et la beauté des images surexposées et filmées en noir et blanc résidait dans la dureté et l'aridité du sertão.

La dureté est ici exprimée par le caractère du personnage de Dora: l'ancienne maîtresse d'école écrit des lettres pour des analphabètes dans l'immense gare de Rio, connue comme la Central do Brasil, où la morale, l'éthique et les valeurs humaines semblent avoir perdu leur importance. "La dure indifférence n'est peut-être que le visage avouable de la nostalgie» (Kristeva, 1988 , p. 20). Cette phrase de Kristeva pourrait nous rapprocher davantage du personnage de Dora. Cette dernière rejette en effet ceux qui lui ressemblent, et c'est ainsi que s'expliquent sa rancune et ses actes mesquins. Elle n'a pas de père non plus, et elle est arrivée à un moment de sa vie où elle n'attend plus aucun geste d'humanité de la part d'autrui. Serait-ce un signe de nostalgie, ou le signe d'une angoisse, d'une frustration venue du passé? Dans la scène finale, Dora regarde avec un monocle la photo d'elle prise avec le gamin devant une image de Padre Cicero, une des figures mythiques de la religion du peuple du sertão. En même temps, en montage parallèle, le gamin regarde la même photo à travers un autre monocle. Ce geste est lié au 
passé par l'entremise de ce cercle de verre, le monóculo, qui permet de voir au fond d'un contenant en plastique la diapositive de la photo. Les personnages sont finalement unis dans ce moment unique où ils se rencontrent véritablement dans la contemplation de la photo. Dora venait juste d'écrire une lettre à l'enfant dans laquelle elle disait ne pas avoir seulement la saudade de son père, mais de tout, de son passé, d'un temps, peut-être, où elle croyait encore à la vérité humaine.

La vérité dans ce film, cette quête majeure, n'est pas dans la finalité de l'histoire, on ne va rien dévoiler, on ne va rien apprendre, malgré le ton moraliste de la fin. S’il y a une vérité, elle est plutôt présente dans les vestiges du passé, et par cela, on retrouve dans les images, avec toutes leurs forces et leurs contradictions, des moments véritables. Des moments montrés par les clients de Dora, par exemple, par leur simplicité, par leur accent, qui retrace les contours régionaux du pays quand ils citent des noms de villes. Ce n'est pas un hasard si le premier personnage apparaissant dans le film est Maria, la femme dont l'histoire fut filmée par Walter Salles dans le documentaire Socorro Nobre, et qui a servi d'inspiration au scénario de Central do Brasil. Ici, elle dicte à Dora une lettre destinée à son amant qui est emprisonné, une référence du réalisateur à l'histoire personnelle de cette femme, révélant les sources documentaires du film. La procession religieuse est également un de ces moments véritables. Comme la profession de Dora, rédactrice de lettres pour des analphabètes, les rituels religieux sont aussi en train de disparaître.

À partir de ces moments nostalgiques, sentimentaux, saudosistas, le film fait un travail de recherche remarquable sur un peuple et un cinéma perdus parmi des milliers d'images stéréotypées qu'il ne renie d'ailleurs pas, les moments évoqués ne leur ressemblant guère.

\section{Le point de vue de l'«individu»:}

\section{les expérimentations narratives dans le documentaire}

Le documentaire a été, dans le cinéma de la Reprise, le genre qui a attiré davantage le public. Il a également obtenu une reconnaissance croissante de la critique, notamment pour la pré- 
sentation de ses nouvelles propositions esthético-narratives ${ }^{8}$ et grâce, entre autres, au faible coût de production des nouvelles technologies.

À la tête de ce boom documentaire au Brésil, on retrouve Eduardo Coutinho, qui non seulement est aujourd'hui le cinéaste le plus influent du pays, selon les cinéastes et les critiques, mais aussi celui à qui l'on doit une esthétique très particulière, très singulière, qui a fait sa marque dans l'histoire récente du documentaire brésilien. Il a commencé sa carrière dans le Cinema Novo. Persécuté par les militaires quand il filmait avec le Parti communiste et les campesinos travaillant dans le sertão nordestin, il reprendra Un homme à abattre (Cabra marcado para morrer), commencé en 1964, seulement vingt ans plus tard (1984), et le portera au rang de documentaire brésilien le plus réputé. Le discours politique est absent dans ses films les plus récents, où l'on constate, par exemple, que Coutinho est à la recherche d'un récit moins linéaire et homogène et qu'il affiche un certain manque d'intérêt pour l'intrigue ou pour un quelconque sujet de recherche, pour se concentrer sur la construction d'une mosaïque narrative des «individualités».

Ce nouveau boom documentaire a accordé la priorité, dans ses grandes lignes, à la qualité esthétique de la production (Fenêtre sur l'âmelJanela da alma, João Jardim et Walter Carvalho, 2001), aux innovations narratives (Le prisonnier des grilles de fer/O prisioneiro da grade de ferro, Paulo Sacramento, 2003), et au traitement des images (Nous qui sommes ici, nous vous attendons/Nós que aqui estamos por vós esperamos, Marcelo Masagão, 1999). Les documentaristes participant à ce renouveau documentaire se souciaient davantage de l'image qu'ils montraient que de l'histoire qu'ils racontaient; en cela réside l'une de ses grandes différences avec le documentaire politique du Cinema Novo.

Le film documentaire se caractérise, comme le cinéma de fiction de la Reprise, par l'attention toute spéciale qui est accordée à la qualité de la production et au raffinement esthétique. Les histoires se révèlent beaucoup plus dans leur style narratif que dans leur potentiel informatif. Lintrigue principale du film ne sert pas tant de fil conducteur ou de point central, elle sert plus 
souvent de prétexte pour dévoiler les personnages, comme Santiago, dans le film du même titre (2006) de João Moreira Salles, ou Estamira, également dans le film du même titre (2004) de Marcos Prado.

Quant à l'esthétique de Coutinho, elle s'est définie et s'est perfectionnée au fil des ans, notamment dans la construction de ses "personnages», de ses cadrages, et dans ses choix narratifs. Il conduit ses interviews comme s'il laminait et affinait le cadre cinématographique. C'est à partir de la composition de ses personnages que l'histoire prend lentement forme.

Dans le cinéma de Coutinho, les témoignages sont uniques et singuliers, et le réalisateur les utilise comme "matière filmique " au service de l'intégrité, de l'entièreté de l'acte de raconter et de fabuler; le principal intérêt de Coutinho, ici, est de ne pas énoncer un fait ni de débattre un sujet en particulier. Dans Fort esprit (Santo Forte, 1999), la religion est un prétexte dont se sert le réalisateur pour amener les interviewés à décrire leur vie quotidienne, à parler de leurs chagrins et de leurs «espoirs». Il en est ainsi dans Babylone 2000 (Babilônia 2000, 2000), où l'attente du nouveau millénaire dans une favela de Rio de Janeiro est un déclencheur servant à faire démarrer une "conversation" (Lins, 2004). Cette stratégie est encore plus poignante dans Master, un bâtiment à Copacabana (Edifício Master, 2002), cet immeuble de Rio de Janeiro étant le seul fil conducteur du film. Il n'existe pas de scénario préétabli, le film ne repose sur aucune intrigue et l'édifice n'est pas le sujet d'une enquête menée par le documentariste. On pourrait penser à la tradition du cinéma direct si le rôle $\mathrm{d}^{\prime}$ intervieweur ${ }^{9}$ du réalisateur n'était pas aussi central dans le film d'après la tradition du cinéma-vérité. Par contre, le film de Coutinho diffère en ceci que le réalisateur y demeure distant, n'en dévoilant pas davantage sur sa personne et encore moins sur son rôle de documentariste. Le but poursuivi par Coutinho est de rassembler un groupe hétérogène de résidents d'un quartier de la classe moyenne brésilienne et de recueillir les faits les plus attrayants et inusités dans les interviews, même si pour cela les interviewés doivent chanter ou danser devant la caméra, et même si leurs témoignages peuvent être coupés au montage final du film s'ils ne possèdent pas de "potentiel cinématographique». 
Xavier parle de la question des rencontres inopinées entre les réalisateurs et les interviewés dans lesquelles les relations se sont individualisées.

Les réalisateurs rencontrent des gens différents : ceux qu'ils interviewent et montrent dans leurs films. Comme Ricardo Dias [Foi, 1999] et Eduardo Coutinho [Fort esprit, 1999] avec les religieux. La rencontre inattendue entre le documentariste, fils d'un banquier, João Moreira Salles et le trafiquant Marcinho VP [dans Nouvelles d'une guerre privée, 1999] constitue l'exemple paradigmatique de ce type de rencontre (2000a, p. 97).

Dans le film de Coutinho, les spectateurs sont à la fois témoins de la rencontre du réalisateur avec la personne interrogée, de même qu'ils se reconnaissent et s'identifient dans les "imprévus» de cette rencontre: la richesse de la parole de chacun. Master est donc un récit entrelacé de va-et-vient, n’utilisant aucune des techniques traditionnelles du scénario, mais plus "proche» du cinéma-vérité que du cinéma direct. Dans ce cinéma, on ne cherche pas les moments de tension ni d'excitation dans lesquels tout s'arrange et où l'on arrive à une catharsis ou à une rédemption collectives. Il n'est donc pas étonnant que Coutinho ait effectué le montage des interviews en ordre plus ou moins chronologique, et qu'il se soit concentré sur les ellipses structurant les témoignages, car le film se tisse et se clôt à chaque énoncé.

On constate une crise de l'histoire en tant qu'instance narrative, une méfiance par rapport à l'énoncé et un désintérêt pour l'intrigue dans les films de Coutinho, en particulier dans Master et, plus tard, dans Jeu de scène (Jogo de cena, 2007), ainsi que dans Amour? (Amor? 2011) de João Jardim: les deux cinéastes ont recours à des acteurs pour interpréter de vraies histoires sous forme d'interviews - dans lesquelles ils soulignent le rôle du fictionnel dans le documentaire.

La préoccupation du film documentaire brésilien contemporain ne réside pas dans la quête d'une histoire à raconter, mais plutôt dans la composition des portraits des individus filmés - qui peuvent être parfois les réalisateurs eux-mêmes, comme dans Un passeport hongrois (Passaporte húngaro, 2003) de Sandra Kogut ou 33 (2002) de Kiko Goifman - afin de les montrer 
sous différents angles, de raconter en se servant de voix multiples et distinctes.

\section{Les cinéastes transnationaux et le cinéma d'auteur en Amérique latine}

Au cours des quinze dernières années, le cinéma brésilien a atteint un niveau de professionnalisme jamais égalé ou même imaginé auparavant, en répondant à la mondialisation du cinéma. Dorénavant, les réalisateurs brésiliens circulent aisément sur tous les marchés cinématographiques mondiaux, et présentent tour à tour des films d'art en Europe, du cinéma engagé en Amérique latine ou du cinéma commercial à l'américaine ou à l'anglaise. À titre d'exemple, pensons aux carrières fulgurantes des réalisateurs Walter Salles (Central do Brasil, 1998 ; Avril brisélAbril despedaçado, 2001 ; Carnets de voyagel Diarios de motocicleta, 2004; Dark Water, 2005; On the Road, 2011), Fernando Meirelles (La Cité de Dieu, 2002; The Constant Gardiner, 2005 ; Blindness, 2008 ; 360, 2011) et, tout récemment, José Padilha (Bus 174/Ônibus 174, 2002; Troupe d'élite, 2007 ; Troupe d'élite 2, 2010) ; ce dernier film est à l'origine du plus gros succès du box-office brésilien et son réalisateur est actuellement convoité par les producteurs de films d'action à gros budget de Hollywood.

Les productions du cinéma brésilien n’ont jamais été aussi internationalisées. Les films de réalisateurs tels que Walter Salles et Fernando Meirelles circulent librement et mondialement dans les festivals internationaux et les marchés du film. Leurs scénarios ont été élaborés dans des ateliers et des laboratoires en Europe et aux États-Unis, et ont également été produits en partenariat avec différents pays. En Amérique du Sud, Salles est devenu un producteur important, socialement et politiquement engagé, pour la nouvelle génération de cinéastes. Meirelles a également développé d'importants partenariats de production qui financent des productions locales, tout en bénéficiant de subventions internationales pour produire ses propres films. Cette génération récente de cinéastes a changé le visage du cinéma brésilien, reflet d'un phénomène touchant aussi le cinéma mondial. Cette nouvelle figure du cinéaste transnational en 
Amérique latine est très éloignée de celle de réalisateurs qui ont bénéficié de la célébrée politique des auteurs des années 1950 et 1960, qui a changé le cinéma européen et influencé la filmographie de Glauber Rocha au Brésil, Tomás Gutiérrez Alea à Cuba et Fernando Solanas en Argentine. La disparition de cette position centrale de l'auteur a pris fin en 1970, lorsque le cinéma autoréférentiel a révélé le symptôme d'une crise, et que les innombrables sources d'information servant à la construction du récit ont décentré celui-ci. Selon András Kovács (2007, p. 355), deux caractéristiques liées au rôle de l'auteur sont essentielles à la modernité dans le cinéma: l'homogénéité du style et un sens de la "réalité objective». S'interroger aujourd'hui sur le rôle de l'auteur dans le cinéma latino-américain n'est pas une réflexion simple. Dans une structure de production cinématographique fragile, les réalisateurs brésiliens et, par extension, les Latino-Américains, sont trop dépendants du crédit d'impôt du gouvernement pour les investisseurs privés et du marché impérialiste de la télévision. Ils n'ont pas de soutien des institutions locales pour produire leurs films. Aucune des structures actuelles n'est soucieuse de l'auteurisme ou de la liberté artistique.

\section{L'hégémonie de l'industrialisation du cinéma brésilien : l'ère Globo Filmes}

Il semble logique de dire, compte tenu des aspects économiques et esthétiques, des impératifs de la production et des critères de performance au box-office, que La Cité de Dieu ${ }^{10}$, réalisé en 2002 par Fernando Meirelles et produit par Walter Salles, a marqué le début d'une nouvelle ère dans le cinéma brésilien, celle de l'industrialisation et du cinéma de genre ou de divertissement.

En 2003, le cinéma brésilien atteignait le sommet de son succès commercial et occupait $25 \%$ du marché interne avec une production totale de 35 nouveaux longs-métrages par année. Pour atteindre ces objectifs, les réalisateurs ont mis en place certaines stratégies narratives et esthétiques afin d'attirer tant le public que le financement privé. Ceux qui ont le mieux su exploiter ces stratégies étaient de jeunes réalisateurs issus du marché publicitaire et de la télévision. À la fin des années 1980 
et au début des années 1990, les telenovelas (séries dramatiques et téléromans) et les annonces publicitaires dépendaient, pour la presque totalité du marché, de techniciens, d'acteurs, de scénaristes et de réalisateurs issus du marché cinématographique ou qui avaient été formés dans les écoles et les universités brésiliennes ${ }^{11}$. Il est intéressant de constater que les films qui précèdent la Reprise, et quelques autres tournés juste après, sont pratiquement des continuations de ce qu'on faisait à la télévision: comme les drames historiques Le quadrille ( $O$ quatrilho, 1994), réalisé par Fábio Barreto, et Quatre jours en septembre ( $O$ que é isso, companheiro? 1997), réalisé par son frère, Bruno Barreto, tous deux mis en nomination aux Oscars pour le meilleur film étranger, ainsi que les films à succès Tieta (Tieta do Agreste, 1996) de Carlos Diegues ou Petit dictionnaire amoureux ( $O$ pequeno dicionário amoroso, 1997) de Sandra Werneck.

Quel genre de films ont-ils produit? Certes, ils ont renoncé, dans la majorité des cas, aux qualités du cinéma d'auteur (raison pour laquelle le Cinema Novo est devenu célèbre) pour privilégier des critères commerciaux. Aucun style en particulier n'a marqué cette période de renaissance du cinéma brésilien allant de 1995 à 2002, mais une large variété de genres et de tendances esthétiques ont dominé les productions, même si l'aspect commercial, qui a influencé l'esthétique de l'époque, demeurait la "priorité » de ces productions.

À contre-courant, le documentaire a vu son public augmenter considérablement dans les salles (Lins et Mesquita 2008), notamment à cause des bas coûts de production de l'audiovisuel numérique, qui ont permis de produire davantage de films. Citons des réalisateurs comme Marcelo Masagão (Nous qui sommes ici, nous vous attendons/Nós que aqui estamos por vós esperamos, 1999), Marcelo Luna et Paulo Caldas (Le rap du petit princelO rap do pequeno principe contra as almas sebosas, 2000), José Padilha (Bus 174/Ônibus 174, 2002), João Moreira Salles (Nelson Freire, 2003; Entractes/Entreatos, 2004) ou encore des cinéastes expérimentaux comme Evaldo Mocarzel (En marge de l'imagelÀ margem da imagem, 2003), Andrea Tonacci (Les monts $d u$ désordre/Serras da desordem, 2006) et Cao Guimarães (Accident/Acidente, 2007). Les images vigoureuses créées par l'ac- 
tuelle production de documentaires, l'inattendu de leurs propos et de leurs stratégies de tournage ${ }^{12}$ ont permis de sauvegarder les qualités expérimentales d'un certain cinéma et du cinéma d'auteur au Brésil, de même qu'ils ont exercé une forte influence sur le cinéma de fiction (Mutum [2007] de Sandra Kogut ; La maison d'AlicelA casa de Alice [2007] de Chico Teixeira), qui reflète les réalités socioéconomiques du pays.

Tous les efforts déployés pour renouveler la cinématographie brésilienne à partir de la Reprise donnent cependant l'impression d'avoir été vains, puisque la production audiovisuelle du cinéma national (comme celui de l'Argentine et du Mexique) semble se diriger vers une plus grande uniformisation. On ne peut que constater dans cette situation l'un des effets de la mondialisation. Par contre, ce processus a entraîné la décentralisation et la régionalisation de la production cinématographique brésilienne (Debs 2004) et la production de films dans le nord et le sud du pays.

Quel type de cinéma s'offre ainsi au public brésilien? Aujourd'hui, le réseau de télévision Globo contrôle un quart de la production des films brésiliens, de la conception à la mise en marché. Entre 1998 et 2003 (Gatti 2005 p. 90), ce réseau a participé de manière directe à la production de 24 films, et sa suprématie s'est finalement cristallisée l'an dernier, quand les films ayant bénéficié de sa participation ont obtenu plus de $90 \%$ des recettes au box-office du cinéma brésilien et ont occupé plus de $20 \%$ du marché total. Comme le réseau de télévision Globo n'est pas considéré comme producteur indépendant, il est le seul à produire des films au Brésil sans aucun investissement ni aide gouvernementale.

Cependant, selon le cinéaste José Joffily (Borges 2007, p. 82), le Brésil n'arrive pas à légiférer sur les radiodiffuseurs, concessionnaires d'un service public. Contrairement à ce que l'on observe dans les autres pays, la télévision brésilienne ne souffre d'aucune sanction légale. À contresens, le réseau de télévision national luimême est en réalité un grand producteur. Partageant le même avis, la cinéaste et productrice Mara Mourão (Borges 2007, p. 82) dénonce le cas de la chaîne TV Globo, conçue selon un modèle unique au monde: il s'agit d'une entreprise qui produit, 
qui distribue et qui diffuse. Cela laisse peu de place à la production indépendante, principalement quand la production privée occupe le terrain du cinéma, qui était jusqu’à présent indépendant et démocratique.

Pour faire une dernière fois référence au Cinema Novo, ce nouveau cinéma de genre ou industriel, qui débute avec le nouveau millénaire au Brésil, est bien loin des idéaux de Glauber Rocha, qui voyait l'alliance du cinéma avec le gouvernement et l'industrialisation de ce secteur comme néfastes à la créativité et à la politique des auteurs latino-américains.

Dans son article « $\mathrm{O}$ avesso dos anos 90 », Xavier synthétise aussi bien le ton de la critique sur le cinéma de la Reprise que le mouvement en soi :

Au centre de la production actuelle, la tendance la plus forte est la reprise de ce qu'il y avait de plus «contractuel» dans le Cinema Novo, mais dans une version encore plus pragmatique de la quête de légitimation mercantile du cinéma brésilien (2001b, p. 5).

Parallèlement à l'ascension du cinéma de la Reprise, la réflexion sur le cinéma brésilien et la critique s'attachant à ce cinéma ont retrouvé une place importante au sein de la pensée sociale du pays, du jamais vu depuis le Cinema Novo. Ce renouveau a été stimulé par la création, il y a dix ans, par des chercheurs et professeurs de cinéma, de la Socine - Société brésilienne des études sur le cinéma et l'audiovisuel. Depuis, de nouvelles approches théoriques et analytiques ont revitalisé la pensée cinématographique et celle-ci a acquis une importance notable dans les débats en sciences humaines au Brésil.

Les textes réunis dans le présent numéro de Cinémas offrent un panorama de cette réflexion théorique brésilienne, une des plus influentes en Amérique latine. Trois points principaux sont abordés dans ces analyses: les choix esthético-narratifs, les films issus de cette période et les thèmes qui y sont privilégiés. L'ensemble de ces cinq articles rédigés par des chercheurs brésiliens respectés propose un bilan et une analyse des nouvelles stratégies et de l'esthétique du cinéma contemporain brésilien, en parcourant les dernières années de la production nationale. 
Pour mener à bien cette entreprise, j'ai invité les théoriciens et les professeurs de cinéma brésilien les plus renommés, ainsi que de jeunes chercheurs, afin d'orienter la discussion sur quelques points privilégiés par le cinéma brésilien au cours des quinze dernières années (1995-2010). Dans leurs articles, ces auteurs examinent la figure du héros et le ressentiment national (Ismail Xavier), les interviews dans le documentaire (Stella Senra), la violence et l'influence de l'esthétique télévisuelle (Gabriela Borges), le dispositif documentaire (Consuelo Lins) et les figures de l'altérité dans le documentaire expérimental (César Guimarães).

J'aimerais remercier les collaborateurs de ce numéro; André Gaudreault, qui a accueilli avec enthousiasme le projet dès le départ; Socine; Stella Senra pour son soutien et ses précieux commentaires; et spécialement, Valérie Martel pour sa lecture patiente et compétente des originaux; je remercie également tous ceux et celles dont la contribution a influencé et facilité la réalisation de ce numéro.

\section{Ryerson University}

\section{NOTES}

1. Troupe d'Élite 2 a fait la plus grosse recette au Brésil; le film qui arrive en deuxième position est Avatar (2009) de James Cameron avec 65,4 millions de dollars américains. Troupe d'Élite 2 est aussi le deuxième film le plus vu dans les salles de cinéma brésiliennes; le premier est Titanic (1997) de James Cameron avec 16 millions de spectateurs. Ces données sont tirées des statistiques de l'Ancine - Agência Nacional de Cinema et du site spécialisé Filme B (http://www.filmeb.com.br).

2. À l'époque, il était connu que certaines salles de cinéma n'investissaient pas non plus dans leur système de son, car les films étrangers, souvent sous-titrés, ne dépendaient pas d'une qualité sonore de haute qualité pour que les dialogues soient parfaitement audibles.

3. J'explique davantage l'influence du mouvement dans le cinéma brésilien dans l'article "Glauber Rocha et l'image-exil de Claro» (Moura 2005).

4. Témoignage recueilli dans le documentaire Que Viva Glauber! d'Aurélio Michiles, TV Cultura (1991).

5. "Até o pior filme brasileiro nos diz mais respeito que o melhor filme estrangeiro ", dans Suplemento Literário OESP, 21 mars 1959, p. 1.

6. Certains théoriciens soulignent que les courts métrages n'ont pas subi le même sort.

7. Xavier explique davantage sa théorie sur le «ressentiment national» dans l'article qu'il publie dans le présent numéro de Cinémas. 
8. Sur les innovations esthético-narratives du documentaire, voir l'article de César Guimarães dans le présent numéro de Cinémas.

9. Sur cette question de l'intervention dans les interviews dans le documentaire, voir l'article de Stella Senra dans le présent numéro de Cinémas.

10. Voir l'article de Gabriela Borges dans le présent numéro de Cinémas sur la question de la violence et sur la controverse causée par La Cité de Dieu.

11. La création des premières écoles de cinéma au Brésil remonte aux années 1960 et les programmes de deuxième et troisième cycles en cinéma ont été créés dans les années 1970, tandis que les cinémathèques de São Paulo et de Rio de Janeiro existaient depuis 1954. Entre autres défenseurs des études cinématographiques au Brésil, figure notamment Salles Gomes, qui a fondé un ciné-club à la Universidade de São Paulo dans les années 1940. Sur l'évolution des études cinématographiques au Brésil, voir Ismail Xavier, "Cinema Studies in Brazil", The SAGE Handbook of Film Studies, vol. 1, Londres, SAGE, 2008, p. 95-100.

12. À ce propos, voir l'article de Consuelo Lins dans le présent numéro de Cinémas.

\section{RÉFÉRENCES BIBLIOGRAPHIQUES}

Bentes 2003 : Ivana Bentes, "The sertáo and the favela in contemporary Brazilian film ", dans Lúcia Nagib (dir.), The New Brazilian Cinema, Londres, I. B. Tauris, 2003, p. 121-138.

Borges 2007: Danielle dos Santos Borges, "A retomada do cinema brasileiro: Uma análise da indústria cinematográfica nacional de 1995 a 2005 ", thèse de doctorat, Barcelona, Universitat Autònoma de Barcelona, 2007.

Debs 2004: Sylvie Debs, "Le cinéma de la Reprise: 1995-2002», Revista Cinémas d'Amérique Latine, $\mathrm{n}^{\circ}$ 12, 2004, p. 10-27.

Deleuze 1985: Gilles Deleuze, Limage-temps, Paris, Minuit, 1985.

Galváo et Souza 1987 : Maria Rita Galvão et Carlos Roberto de Souza, «Le parlant et les tentatives industrielles: années trente, quarante, cinquante», dans Paulo Antônio Paranaguá (dir.), Le cinéma brésilien, Paris, Centre Georges Pompidou, 1987, p. 67-89.

Gatti 2005 : André Piero Gatti, «Distribuição e exibição na indústria cinematográfica brasileira (1993-2003)", thèse de doctorat, Campinas, Universidade Estadual de Campinas, 2005.

Hamburguer 2005 : Esther Hamburguer, «Políticas da representação : ficção e documentário em Onibus 174 ", dans Maria Dora Mourão et Amir Labaki (dir.), O cinema do real, São Paulo, Cosac e Naify, 2005, p. 196-215.

Kovács 2007: András Bálint Kovács, Screening modernism: European Art Cinema, 1950-1980, Chicago, The University of Chicago Press, 2007.

Kristeva 1988: Julia Kristeva, Etrangers à nous-mêmes, Paris, Gallimard, 1988.

Lins 2004: Consuelo Lins, $O$ documentário de Eduardo Coutinho. Televisão, cinema e vídeo, Rio de Janeiro, Jorge Zahar Editor, 2004.

Lins et Mesquita 2008: Consuelo Lins et Cláudia Mesquita, Filmar o real, Rio de Janeiro, Jorge Zahar Editor, 2008.

Moura 2005: Hudson Moura, "Glauber Rocha et l'image-exil de Claro », Cinémas, $\mathrm{n}^{\circ} 15,2005$, p. 81-103.

Moura 2008: Hudson Moura, "Les rencontres inattendues et interculturelles dans les cinémas brésilien et québécois", dans Jean Fisette et Licia Soares de Souza (dir.), actes du colloque Échanges Brésil-Québec. Les savoirs en mouvement, CD-Rom, 2008, p. 1-11. 
Nagib 2002: Lúcia Nagib (dir.), O cinema da retomada - Depoimentos de 90 cineastas dos anos 90, São Paulo, 34, 2002.

Nagib 2007 : Lúcia Nagib, Brazil on Screen, Londres, I. B. Tauris, 2007.

Oricchio 2003: Luiz Zanin Oricchio, Cinema de Novo: Um balanço crítico da retomada, São Paulo, Estação Liberdade, 2003.

Pape 2004 : Lygia Pape, "Cinema Marginal», dans Portal Brasileiro de Cinema, 2004. Accès en janvier 2011 : http://www.heco.com.br/marginal/ontem $\% 20$ hoje\% 20amanha/04_01_10.php

Salles Gomes 1987 : Paulo Emílio Salles Gomes, "Trajectoire dans le sousdéveloppement" [1973], dans Paulo Antônio Paranaguá (dir.), Le cinéma brésilien, Paris, Centre Georges Pompidou, 1987, p. 11-19.

Senra 2002 : Stella Senra, "Amélia, dependência de dois mundos que se estranham ", Sinopse, ${ }^{\circ}$ 8, 2002, p. 38-41.

Senra 2006 : Stella Senra, "Pelo retrovisor : a nostalgia em Cinema, aspirinas e urubus, de Marcelo Gomes » Sinopse, n 11, 2006, p. 97-99.

Xavier 1983: Ismail Xavier, Sertão Mar: Glauber Rocha e a estética da fome, São Paulo, Brasiliense, 1983.

Xavier 1993 : Ismail Xavier, Alegorias do subdesenvolvimento: Cinema Novo, Tropicalismo e Cinema Marginal, São Paulo, Brasiliense, 1993.

Xavier 2000a : Ismail Xavier, "O cinema brasileiro dos anos 90 ", Praga - Estudos marxistas, $\mathrm{n}^{\circ}$ 9, 2000, p. 97-138.

Xavier 2000b : Ismail Xavier, "Encontros inesperados. Entrevista a Mario Sérgio Conti ", Folha de São Paulo, 3 décembre 2000, p. 8-9.

Xavier 2001a: Ismail Xavier, "A figura do ressentimento no cinema brasileiro dos anos 90 ", dans José Gatti et al. (dir.), Estudos de cinema 2000: Socine, Porto Alegre, Sulina, 2001.

Xavier 2001b : Ismail Xavier, "O avesso dos anos 90 », Folha de São Paulo, 10 juin 2001, p. 4-5.

Xavier 2001c: Ismail Xavier, O cinema brasileiro moderno, São Paulo, Paz e Terra, 2001.

Xavier 2002 : Ismail Xavier, "Microcosmo em celulóide", Folha de São Paulo, $1^{\text {er }}$ décembre 2002, p. 10-11.

Xavier 2003 : Ismail Xavier, "Brazilian cinema in the 1990s: The unexpected encounter and the resentful character", dans Lúcia Nagib (dir.), The New Brazilian Cinema, Londres, I. B. Tauris, 2003, p. 39-64.

Xavier 2009: Ismail Xavier, Glauber Rocha et l'esthétique de la faim [1983], traduction et présentation par Sylvie Debs, Paris, L'Harmattan, 2009. 